\title{
5. Ueber den Mittelwert, der durch die kleinste Abweichung definiert wird.
}

\author{
Von Mitio Nagumo
}

(Eingegangen am 27. Dezember, 1932)

\section{$\$ 1$.}

Das arithmetische Mittel von $n$ reellen Zahlen $\xi_{1}, \xi_{2}, \ldots, \xi_{\text {u }}$ kann als der jenige Wert von $x$ aufgefasst werden, für den die Funktion $\sum_{i=1}^{n}\left(x-\xi_{i}\right)^{2}$ das Minimum annimmt. Den Begriff des Mittelwertes können wir also folgendermassen erweitern: Hier verstehen wir unter dem Mittelwert $M_{\varphi}\left(\xi_{1}, \xi_{2}, \ldots, \xi_{i}\right)$ von $n$ reellen Zahlen $\xi_{1}, \xi_{2}, \ldots, \xi_{n}$ denjenigen Wert von $x$, für den die Funktion $\sum_{i=1}^{n} \varphi\left(\left|x-\xi_{i}\right|\right)$ das Minimum annimmt, wobei $\varphi(x)$ eine geeignete stetige stets wachsende Funktion von $x \geqq 0$ ist, die wir nachher genamer bestimmen wollen.

Satz I. Dafür, dass die Funktion $\sum_{i=1}^{n} \varphi\left(\left|x-\xi_{i}\right|\right)$ für einen und nur einen Wert von $x$ das (absolute) Winimum annehme, ist es notwendig und hinieichend, dass $\varphi(x)$ eine cht konvere Funktion von $x \geqq 0$ ist.

Wenn $\varphi(x)$ eine ccht konvexe Funktion ist, so hängt der Mittelwest $M_{\varphi}\left(\vec{\xi}_{1}, \xi_{2}, \ldots, \xi_{n}\right)$ von $\left(\xi_{1}, \xi_{2}, \ldots, \xi_{n}\right)$ stetig $a b$.

Beweis: Es sei $\varphi(x)$ eine echt konvexe (stets wachsende stetige) Funktion. Dann ist $\sum_{i=1}^{n} \varphi\left(\left|x-\xi_{i}\right|\right)$ auch eine echt konvexe Funktion. Man setze $\alpha=\operatorname{Min}_{1 \leq i \leq n}\left(\xi_{i}\right)$ und $\beta=\underset{1 \leqq i \leqq n}{\operatorname{Max}}\left(\xi_{i}\right) . \quad \sum_{i=1}^{n} \varphi\left(x-\xi_{i} \mid\right)$ wächst dann immer grösser ausserhalb des Intervalles $\alpha \leqq v \leqq \beta$, wenn $x$ sich von $\alpha$ oder $\beta$ entfernt. Es gibt also einen und nur einen Wert von $x$, für den $\sum_{i=1}^{n} \varphi\left(\left|x-\xi_{i}\right|\right)$ das Minimum annimmt; und dieser Wert von $x$ liegt sogar in. $\alpha \leqq x \leqq \beta$.

Um die Notwendigkeit der Bedingung zu beweisen, betrachten wir den Fall $n=2, \xi_{1}=-a, \xi_{2}=a, a>0$. $x_{0}$ sei derjenige Wert von $x$, für den. $\varphi(|x+a|)+\varphi(|x-a|)$ das Minimum annimmt. Aus der Relation

$$
\sum_{i=1}^{2} \varphi\left(\left|x_{0}-\xi_{i}\right|\right)=\varphi\left(\left|x_{0}+a\right|\right)+\varphi\left(\left|x_{0}-a\right|\right)=\varphi\left(\left|a+x_{0}\right|\right)+\varphi\left(\left|a-x_{0}\right|\right)
$$


folgt aber, dass $\varphi(|x+a|)+\varphi(|x-a|)$ denselben Wert für $x=x_{0}$ and $x=-x_{0}$ erhält. Wegen der Eindeutigkeit des Minimums gilt also $x_{0}=0$. Nach der Definition des Minimums besteht also für $a>x>0$

$$
\varphi(|x+a|)+\varphi(x-a \mid)=\varphi(a+x)+\varphi(a-x)>2 \varphi(a),
$$

oder für zwei beliebige von einander verschiedene positive Zahlen $x_{1}$ und $x_{2}$

$$
\frac{\varphi\left(x_{1}\right)+\varphi\left(x_{2}\right)}{2}>\varphi\left(\frac{x_{1}+x_{2}}{2}\right) .
$$

$\varphi(x)$ ist also eine echt konvexe Funktion von $x$.

Um den letzten Teil von Satz 1 zu beweisen, schicken wir den folgenden Hilfssatz voraus, der auch für eine spätere Erörterung nützlich ist.

Hilfssatz. Es sei $f\left(x, \alpha_{1}, \alpha_{2}, \ldots, \alpha_{2}\right)$ eine stetige Funltion von $(x$, $\left.\alpha_{1}, \alpha_{2}, \ldots, \alpha_{p}\right)$ im Bereich $x_{0} \leqq x \leqq x_{1}, \alpha_{\nu}<\alpha_{\nu}<b_{\nu}$, die für jedes $\left(\alpha_{1}, \alpha_{2}, \ldots\right.$, $\alpha_{n}$ ) an einer und nur an einer Stelle im Intervalle $x_{i} \leqq x \leqq x_{1}$, nämlich an $x=\xi$, das Minimum annimmt. Dann hängt $\xi$ von $\left(\alpha_{1}, \alpha_{2}, \ldots, \alpha_{i}\right)$ stetig $a b$.

Beweis von dem Hilfssatz: Wir nehmen an, dass für $\alpha_{v}=\alpha_{v}^{(0)}\left(\alpha_{v}\right.$ $\left\langle\alpha_{\nu}^{(0)}<b_{\nu}\right)$ die Funktion $\xi_{0}\left(\alpha_{1}, \alpha_{2}, \ldots, \alpha_{1}\right)$ nicht stetig wäre. Dann gibt es eine gegen $\alpha_{v}=\alpha_{v}^{(0)}$ konvergierende Folge $\alpha_{v}^{(n)}$ derart, dass $\xi\left(\alpha_{1}^{(n)}, \ldots \ldots\right.$, $\left.\alpha_{p^{\prime}}^{(n)}\right)=\xi_{n}$ gegen einen von $\xi\left(\alpha_{1}^{(0)}, \ldots, \alpha_{p^{\prime}}^{(0)}\right)=\xi_{0}$ verschiedenen Wert von $x$, etwa $x=\xi^{\prime}$, konvergiert. Da $f\left(x, \alpha_{1}, \ldots, \alpha_{p}\right)$ nur für einen Wert von $x$ in $x_{0} \leqq x \leqq x_{1}$ das Minimum annimmt, so gilt

und

$$
f\left(\xi_{0}, \alpha_{1}^{(0)}, \ldots, \alpha_{p}^{(0)}\right)<f\left(\xi^{\prime}, \alpha_{1}^{(1)}, \ldots, \alpha_{p}^{(0)}\right),
$$

Aus der zweiten Ungleichung folgt für $n \rightarrow \infty$

$$
f\left(\xi_{0}, \alpha_{1}^{(0)}, \ldots, \alpha_{p}^{(0)}\right) \geqq f\left(\xi^{\prime}, \alpha_{1}^{(0)}, \ldots, \alpha_{p}^{(0)}\right)
$$

im Widerspruch mit der ersten Ungleichung. Damit ist der Beweis von dem Hilfssatz erledigt.

Nun beweisen wir den letzten Teil von Satz 1. Nach dem ersten Teil des Beweises von Satz 1 nimmt die Funktion $\sum_{i=0}^{n} \varphi\left(\left|x-\xi_{i}\right|\right)$ das Minimum für einen und nur einen Wert von $x$ im Intervalle $x_{0} \leqq x_{n_{1}}$ $\left(x_{0}<\xi_{\nu}<x_{1}\right)$ an. $\sum_{i=1}^{\prime \prime} \varphi\left(\left|x-\xi_{i}\right|\right)$ ist auch eine stetige Funktion von $\left(x, \xi_{1}\right.$, $\left.\ldots, \xi_{n}\right)$. Nach dem Hilfssatz ist also $M_{\varphi}\left(\xi_{1}, \ldots, \xi_{n}\right)$ eine stetige Funktion von $\left(\xi_{1}, \ldots, \xi_{n}\right)$. W. z. b. W. 
$\$ 2$.

Wir legen voln jetzt an fest, dass $\varphi(x)$ eine stetige stets wachsende echt konvexe Funlition won $x \geqq 0$ ist und $\varphi(0)=0$. Die Funktion $M_{\varphi}\left(\xi_{1}\right.$, $\ldots, \xi_{n}$ ) hat auch dieselbe Bedeutung wie vorher.

Satz 2. Dafüi dass der Wittelwert $I_{\varphi}\left(\xi_{1}, \ldots, \xi_{n}\right)$ der Relation

$$
M_{\varphi}\left(k \xi_{1} . k \xi_{2}, \quad ., k \xi_{n}\right)=k H_{\varphi}\left(\xi_{1}, \xi_{2}, \ldots, \xi_{n}\right)
$$

für eine beliebige reelle Konstante $k$ genüge, ist es notwendig und hinreichend, dass $\varphi(x)$ won der Form $\varphi(x)=e^{a}(x \geqq 0)$ ist, wobei $\alpha>1, c>0$.

Beweis: Die Hinlänglichkeit der Bedingung kann man leicht beweisen. Denn die beiden Funktionen, $\sum_{i=1}^{n} c|x-\xi|^{a}$ and $\sum_{i=1}^{n} c\left|k x-k \xi_{i}\right|^{a}$, $(c>0, \alpha>1)$, nehmen die Minimumwerte gleichzeitig für denselben Wert von $x$ an.

Um die Notwendigkeit der Bedingung zu beweisen, betrachten wir uns die Funktion $\mu \varphi(|x-a|)+(1-\mu) \varphi(|x-b|),(0 \leqq \mu \leqq 1)$. Diese echt konvexe Funktion nimmt das Minimum genau für einen Wert von $x$ an, den wir mit $M_{\varphi}(a, b ; \mu)$ ausdrücken. Wie man es leicht sieht, gilt $a \leqq M_{\varphi}(a, b ; \mu) \leqq b(0 \leqq \mu \leqq 1)$. Nach dem Hilfssatz ist $M_{\varphi}(a, b ; \mu)$ eine stetige Funktion von $(a, b, \mu)$. Aus den Definitionen von $M_{\varphi}\left(\xi_{1}, \xi_{2}, \ldots\right.$, $\left.\xi_{n}\right)$ und $M_{\varphi}(a, b ; \mu)$ kann man auch leicht schliessen, das

$$
M_{\varphi}(\underbrace{a, a, \quad, \quad, b, b, \ldots, b}_{m-\text { mal }})=M_{\varphi}\left(a, b ; \frac{m}{n}\right) \text {. }
$$

Es gilt

(i)

$$
M_{\varphi}\left(k(t, k b ; \mu)=k H_{\varphi}(a . b ; \mu)(0 \leqq \mu \leqq 1 .\right.
$$

Denn nach der Voraussetzung gilt

$$
M_{\varphi}(\underbrace{k a, k a, \ldots, k a}_{m-\text { mal }}, \frac{k b, k b, \ldots, k b)}{(n-m)-\mathrm{mal}}=k M_{\varphi}(\underbrace{(a, \ldots, a}_{m-\text { mal }}, a, \underbrace{b, \ldots, b}_{(n-m)-\text { mal }}) .
$$

Also

$$
M_{\varphi}\left(k c, k b ; \frac{m}{n}\right)=k M_{\varphi}\left(a, b ; \frac{m}{n}\right)
$$

Lässt mau $\frac{m}{n}$ gegen $\mu(0 \leqq \mu \leqq 1)$ konvergieren, so erhält man die Relation. (i). Die Relation (i) lautet für die Funktion $\mu \varphi(|x-a|)$ $+(1-\mu) \varphi(|x-b|)$, wenn $\operatorname{man} a=0, b=1, M_{\varphi}(0.1 ; \mu)=X$ setzt,

$$
\left\{\begin{array}{l}
D_{-}\{\mu \varphi(|k X|)+(1-\mu) \varphi(|k(1-X)|)\} \leqq 0 \\
D_{+}\{\mu \varphi(k X \mid)+(1-\mu) \varphi(|k(1-X)|)\} \geqq 0
\end{array}\right.
$$

wobei $D_{-}$die linksseitige und $D_{+}$die rechtsseitige Derivierte nach $X$ 
bedentet. $\mathrm{Da} \mu_{\varphi}(0,1 ; \mu)$ eine stetige Funktion von $\mu(0 \leqq \mu \leqq 1)$ ist, und $M_{\varphi}(0,1 ; 0)=1, M_{\varphi}(0,1 ; 1)=0$, so gibt es für jedes $X, 0<X<1$, ein $\mu(X)(0<\mu(X)<1)$ derart, dass $X=M_{\varphi}(0,1 ; \mu(X))$. Wir bekommen also (ii) für $K>0,0<X<1$

und

$$
\frac{\mu(X)}{1-\mu(X)} D_{-} \varphi(k X) \leqq D_{+} \varphi(k(1-X))
$$

$$
\frac{\mu(X)}{1-\mu(X)} D_{+} \varphi(k X) \geqq D_{-} \varphi(k(1-X)) \text {. }
$$

Weil für die konvexe Funktion $\varphi(x)$ die Relation $\lim _{x \rightarrow x_{0}+0} D_{ \pm} \varphi(x)=\nu_{+} \varphi\left(x_{0}\right)$ $\left(c_{0} \geqq 0\right)$ besteht, so gilt für $k \rightarrow k_{0}+0 \quad\left(k_{0}>0\right)$

$$
\frac{\mu(X)}{1-\mu(X)} D_{+} \varphi\left(k_{0} X\right) \leqq D_{+} \varphi\left(k_{v}(1-X)\right),
$$

und

$$
\frac{\mu(X)}{1-\mu(X)} D_{+} \varphi\left(k_{0} X\right) \geqq D_{+} \varphi\left(k_{0}(1-X)\right),
$$

folglich

$$
D_{+} \varphi\left(k_{0}(1-X)\right)=D_{+} \varphi\left(k_{0} X\right) \frac{\mu(X)}{1-\mu(X)} .
$$

Für beliebige positive Zahlen $u$ und $v$ gibt es reelle Zahlen $X(0<X<1)$ and $k_{0}>0$, sodass $u=k, X$ und $v=\frac{1-X}{X}$. Also für beliebige positive Zahlen $w$ und $v$

$$
D_{+} \varphi(u, v)=D_{+} \varphi(u) \Psi(v) \quad\left(\psi(v)=\frac{\mu(X(v))}{1-\mu(X(v))}\right) .
$$

Da $D_{+} \varphi(x)$ monoton wächst, so ist $D_{+} \varphi(x)$ mindestens für einen positiven Wert von $x$ stetig. Aus der Funktionalgleichung kann man daher leicht schliessen, dass $D_{+} \varphi(u)$ für $u>0$ immer stetig ist. Folglich $D_{+} \varphi(x)=D_{-} \varphi(x)=\frac{d}{d x} \varphi(x)=\varphi^{\prime}(x)$ für $x>0 . \quad$ Also

$$
\varphi^{\prime}(u v)=\varphi^{\prime}(u) \psi(v) \text {. }
$$

Setzt man $u=1$, dann $\varphi^{\prime}(v)=\varphi^{\prime}(1) \psi(v)$. Folglich

$$
\psi(u v)=\psi(u) \psi(v)
$$

Da $\psi(u)$ eine stetige positive Funktion ist, (denn $\varphi^{\prime}(u)>0$ und $\psi(u)=$ $\frac{\varphi^{\prime}(u)}{\varphi^{\prime}(1)}$ für $\left.u>0\right)$, gilt $\psi(u)=u^{p}(u>0, p=$ Konst. $) \quad$ Daher $\varphi^{\prime}(u)=\varphi^{\prime}(1) u^{\prime \prime}$ $\varphi^{\prime}(1)$
$(u>0)$, und folglich $\quad \varphi(u)=C_{u^{a}} \quad\left(\alpha=p+1, C=\frac{\varphi^{\prime}(1)}{p+1}\right)$.

Aus der Bedingung, dass $\varphi(x)$ eine stets wachsende echt konvexe Funktion von $x \geqq 0$ ist, folgt $C>0, \alpha>1$. W. z. b. W.

Göttingen, den 5. December 1932. 